\title{
Novel PT-invariant Solutions For a Large Number of Real Nonlinear Equations
}

\author{
Avinash Khare \\ Physics Department, Savitribai Phule Pune University \\ Pune, India 411007 \\ Avadh Saxena
}

Theoretical Division and Center for Nonlinear Studies, Los Alamos National Laboratory, Los Alamos, NM 87545, USA

\begin{abstract}
:
For a large number of real nonlinear equations, either continuous or discrete, integrable or nonintegrable, we show that whenever a real nonlinear equation admits a solution in terms of $\operatorname{sech} x$, it also admits solutions in terms of the PT-invariant combinations $\operatorname{sech} x \pm i \tanh x$. Further, for a number of real nonlinear equations we show that whenever a nonlinear equation admits a solution in terms $\operatorname{sech}^{2} x$, it also admits solutions in terms of the PT-invariant combinations $\operatorname{sech}^{2} x \pm i \operatorname{sech} x \tanh x$. Besides, we show that similar results are also true in the periodic case involving Jacobi elliptic functions.
\end{abstract}




\section{Introduction}

Nonlinear equations are playing an increasingly important role in several areas of science in general and physics in particular [1]. One of the major problems with these equations is the lack of a superposition principle. It may be thus desirable to explicitly obtain as many solutions of a given nonlinear equation as possible. It is, however, possible that auto-Bäcklund transformations may also provide such solutions in the case of integrable [2] and non-integrable [3] models. Thus, if we can find some general results about the existence of solutions to a nonlinear equation, that would be invaluable, at least in the case of nonintegrable equations. In this context it is worth recalling that some time ago we $[4,5]$ had shown (through a number of examples) that if a nonlinear equation admits a periodic solution in terms of Jacobi elliptic functions $\operatorname{dn}(x, m)$ and $\operatorname{cn}(x, m)$, then it will also admit solutions in terms of $\operatorname{dn}(x, m) \pm \sqrt{m} \operatorname{cn}(x, m)$, where $m$ is the modulus of the elliptic function [6]. Further, in the same papers $[4,5]$, we also showed (again through several examples) that if a nonlinear equation admits a solution in terms of $\operatorname{dn}^{2}(x, m)$, then it will also admit solutions in terms of $\operatorname{dn}^{2}(x, m) \pm \sqrt{m} \operatorname{cn}(x, m) \operatorname{dn}(x, m)$.

The purpose of this paper is to propose general results about the existence of new solutions to real nonlinear equations, integrable or nonintegrable, continuous or discrete through the idea of parity-time reversal or PT symmetry. It may be noted here that in the last 15 years or so the idea of PT symmetry [7] has given us new insights. In quantum mechanics it has been shown that even if a Hamiltonian is not hermitian but if it is PT-invariant, then the energy eigenvalues are still real in case the PT symmetry is not broken spontaneously. Further, there has been a tremendous growth in the number of studies of open systems which are specially balanced by PT symmetry $[8,9,10]$ in several PT-invariant open systems bearing both loss and gain. In particular, many researchers have obtained soliton solutions which have been shown to be stable within a certain parameter range $[11,12,13]$.

In this paper we highlight one more novel aspect of PT symmetry. Specifically, we obtain new PTinvariant solutions of several real nonlinear equations. Our strategy will be to start with known real solutions and then make Ansätze for complex PT-invariant solutions and obtain conditions under which the Ansätze are valid. We show, through several examples, that whenever a real nonlinear equation, either continuous or discrete, integrable or nonintegrable, admits a solution in terms of $\operatorname{sech} x$, then it 
will necessarily also admit solutions in terms of the PT-invariant combinations $\operatorname{sech} x \pm i \tanh x$. We also generalize these results to the periodic case and show that whenever a nonlinear equation admits a solution in terms of $\operatorname{dn}(x, m)$ [or $\mathrm{cn}(x, m)]$, then it will necessarily also admit solutions in terms of the PT-invariant combinations $\operatorname{dn}(x, m) \pm i \sqrt{m} \operatorname{sn}(x, m)[\operatorname{or} \operatorname{cn}(x, m) \pm i \operatorname{sn}(x, m)]$.

In addition, we also show through several examples that whenever a real nonlinear equation admits a solution in terms of $\operatorname{sech}^{2} x$, then it will also admit solutions in terms of $\operatorname{sech}^{2} x \pm i \operatorname{sech} x \tanh x$. We also generalize these results to the periodic case and show that whenever a real nonlinear equation admits a solution in terms of $\operatorname{dn}^{2}(x, m)$, then it will necessarily also admit solutions in terms of $\operatorname{dn}^{2}(x, m) \pm$ $i m \operatorname{sn}(x, m) \operatorname{cn}(x, m)$ as well as $\mathrm{dn}^{2}(x, m) \pm i \sqrt{m} \operatorname{sn}(x, m) \operatorname{dn}(x, m)$. These results may find physical realization in coupled optical waveguides among other applications [14]. It is worth pointing out that some of the equations considered here have also been considered in their PT-symmetric deformed version [15].

\section{Solutions in Terms of $\operatorname{sech} x \pm i \tanh x$ and Their Periodic Generalization}

We now discuss four examples, two from continuum field theories and two from the discrete case where $\operatorname{sech} x$ is a known solution and in all the four cases we obtain new PT-invariant solutions in terms of $\operatorname{sech} x \pm i \tanh x$ and also periodic PT-invariant solutions in terms of $\operatorname{dn}(x, m) \pm i \operatorname{sn}(x, m)$ as well as $\operatorname{cn}(x, m) \pm i \operatorname{sn}(x, m)$.

\section{$2.1 \quad \phi^{4}$ Field Theory}

The $\phi^{4}$ field theory arises in several areas of physics [16] including second order phase transitions. The field equation for the $\phi^{2}-\phi^{4}$ field theory is given by

$$
\phi_{x x}=a \phi+b \phi^{3}
$$

In case $b<0$, one of the well known solutions to this equation is

$$
\phi=A \operatorname{sech}[\beta x]
$$

provided

$$
b A^{2}=-2 \beta^{2}, \quad a=\beta^{2} .
$$


Remarkably, even

$$
\phi=A \operatorname{sech}(\beta x) \pm i B \tanh (\beta x)
$$

is an exact PT-invariant solution of Eq. (1) provided

$$
B= \pm A, \quad 2 b A^{2}=-\beta^{2}, \quad a=-(1 / 2) \beta^{2} .
$$

Further, as we now show, such PT-invariant solutions also exist in the periodic case. Let us first note that one of the exact, periodic solutions to the $\phi^{4}$ Eq. (1) is [17]

$$
\phi=A \operatorname{dn}(\beta x, m),
$$

provided

$$
b A^{2}=-2 \beta^{2}, \quad a=(2-m) \beta^{2} .
$$

Further, the same model (1) is known to admit another periodic solution

$$
\phi=A \sqrt{m} \operatorname{cn}(\beta x, m)
$$

provided

$$
b A^{2}=-2 \beta^{2}, \quad a=(2 m-1) \beta^{2} .
$$

Remarkably, we find that the same model also admits the PT-invariant periodic solution

$$
\phi=A \operatorname{dn}(\beta x, m)+i B \sqrt{m} \operatorname{sn}(\beta x, m)
$$

provided

$$
B= \pm A, \quad 2 b A^{2}=-\beta^{2}, \quad a=-\frac{2 m-1}{2} \beta^{2} .
$$

Further, the same model also admits another PT-invariant solution

$$
\phi=A \sqrt{m} \operatorname{cn}[\beta x, m]+i B \sqrt{m} \operatorname{sn}[\beta x, m]
$$

provided

$$
B= \pm A, \quad 2 b A^{2}=-\beta^{2}, \quad a=-\frac{2-m}{2} \beta^{2} .
$$




\section{$2.2 \mathrm{mKdV}$ Equation}

We first discuss the celebrated modified Korteweg-de Vries (mKdV) equation

$$
u_{t}+u_{x x x}+6 u^{2} u_{x}=0
$$

which is a well known integrable equation having application in several areas [16]. It is well known [16] that

$$
u=A \operatorname{sech}[\beta(x-v t)
$$

is an exact solution of Eq. (14) provided

$$
A^{2}=\beta^{2}, v=\beta^{2}
$$

Remarkably, even

$$
u=A \operatorname{sech}[\beta(x-v t)] \pm i B \tanh [\beta(x-v t)]
$$

is also an exact PT-invariant solution to the mKdV Eq. (14) provided

$$
B= \pm A, \quad A^{2}=4 \beta^{2}, \quad v=-(1 / 2) \beta^{2} .
$$

Even more remarkable, such PT-invariant solutions also exist in the periodic case. For example, it is well known that one of the exact, periodic solutions to the mKdV Eq. (14) is [16]

$$
u=A \operatorname{dn}[\beta(x-v t), m]
$$

provided

$$
A^{2}=\beta^{2}, \quad v=(2-m) \beta^{2} .
$$

Another periodic solution to the mKdV Eq. (14) is

$$
u=A \sqrt{m} \operatorname{cn}[\beta(x-v t), m],
$$

provided

$$
A^{2}=\beta^{2}, \quad v=(2 m-1) \beta^{2} .
$$


Remarkably, even

$$
u=A \operatorname{dn}[\beta(x-v t), m]+i B \sqrt{m} \operatorname{sn}[\beta(x-v t), m]
$$

is an exact PT-invariant solution to the $\mathrm{mKdV}$ Eq. (14) provided

$$
B= \pm A, \quad A^{2}=4 \beta^{2}, \quad v=-\frac{(2 m-1)}{2} \beta^{2} .
$$

We thus have two new periodic solutions of mKdV Eq. (14) depending on whether $B=A$ or $B=-A$.

Further, even

$$
u=A \sqrt{m} \operatorname{cn}[\beta(x-v t), m]+i B \sqrt{m} \operatorname{sn}[\beta(x-v t), m],
$$

is an exact PT-invariant solution of the mKdV Eq. (14) provided

$$
B= \pm A, \quad A^{2}=4 \beta^{2}, \quad v=-\frac{(2-m)}{2} \beta^{2} .
$$

\subsection{Discrete $\phi^{4}$ Equation}

We now discuss two discrete models and show that both these models also admit PT-invariant solutions. Let us first consider the discrete $\phi^{4}$ equation

$$
\frac{1}{h^{2}}\left[\phi_{n+1}+\phi_{n-1}-2 \phi_{n}\right]+a \phi_{n}-\frac{\lambda}{2} \phi_{n}^{2}\left[\phi_{n+1}+\phi_{n-1}\right]=0
$$

It is well known that the Eq. (27) admits an exact solution [18]

$$
\phi_{n}=A \operatorname{sech}(\beta n),
$$

provided

$$
A^{2}=-\frac{2 \sinh ^{2}(\beta)}{h^{2} \lambda}, a h^{2}=-4 \sinh ^{2}(\beta / 2) .
$$

Remarkably, the same model also admits a PT-invariant periodic solution

$$
\phi_{n}=A \operatorname{sech}(\beta n) \pm i B \tanh (\beta n),
$$

provided

$$
B= \pm A, \quad A^{2}=-\frac{2 \tanh ^{2}(\beta / 2)}{h^{2} \lambda}, a h^{2}=2 \tanh ^{2}(\beta / 2)
$$


Besides, the same model also has novel, PT-invariant periodic solutions. Let us first note that a well known exact periodic solution [18] to the Eq. (27) is

$$
\phi_{n}=A \operatorname{dn}(\beta n, m) \text {, }
$$

provided

$$
A^{2} \operatorname{cs}^{2}(\beta, m)=-\frac{2}{h^{2} \lambda}, \quad a h^{2}=2\left[1-\frac{d n(\beta, m)}{\operatorname{cn}^{2}(\beta, m)}\right]
$$

where $\operatorname{cs}(x, m)=\operatorname{cn}(x, m) / \operatorname{sn}(x, m)$. Further, the same model $(27)$ is known to admit another periodic solution

$$
\phi_{n}=A \sqrt{m} \operatorname{cn}(\beta n, m],
$$

provided

$$
A^{2} \mathrm{ds}^{2}(\beta, m)=-\frac{2}{h^{2} \lambda}, \quad a h^{2}=2\left[1-\frac{c n(\beta, m)}{\operatorname{dn}^{2}(\beta, m)}\right],
$$

where $\operatorname{ds}(x, m)=\operatorname{dn}(x, m) / \operatorname{sn}(x, m)$.

We find that the same model also admits the PT-invariant periodic solution

$$
\phi_{n}=A \operatorname{dn}(\beta n, m)+i B \sqrt{m} \operatorname{sn}(\beta n, m),
$$

provided

$$
B= \pm A, \quad A^{2}[\operatorname{cs}(\beta, m)+\mathrm{ns}(\beta, m)]^{2}=-\frac{2}{h^{2} \lambda}, \quad a h^{2}=2\left[1-\frac{2 \operatorname{dn}(\beta, m)}{1+\operatorname{cn}(\beta, m)}\right]
$$

Further, the same model also admits another PT-invariant solution

$$
\phi_{n}=A \sqrt{m} \operatorname{cn}(\beta n, m)+i B \sqrt{m} \operatorname{sn}(\beta n, m),
$$

provided

$$
B= \pm A, \quad A^{2}[\operatorname{ds}(\beta, m)+\operatorname{ns}(\beta, m)]^{2}=-\frac{2}{h^{2} \lambda}, \quad a h^{2}=2\left[1-\frac{2 \operatorname{cn}(\beta, m)}{1+\operatorname{dn}(\beta, m)}\right] .
$$

While deriving results in this and the next subsection, we have made use of several not so well known identities satisfied by the Jacobi elliptic functions [19]. 


\subsection{Discrete mKdV Equation}

Let us consider the discrete $\mathrm{mKdV}$ equation

$$
\frac{d u_{n}}{d t}+\alpha\left(u_{n+1}-u_{n-1}\right)+\lambda u_{n}^{2}\left(u_{n+1}-u_{n-1}\right)=0 .
$$

It is well known that this model has an exact hyperbolic soliton solution [20]

$$
u_{n}=A \operatorname{sech}[\beta(n-v t)]
$$

provided

$$
\lambda A^{2}=\alpha \sinh ^{2}(\beta), \quad \beta v=2 \alpha \sinh (\beta) .
$$

We find that this model also admits the PT-invariant solution

$$
u_{n}=A \operatorname{sech}(\beta n) \pm i B \tanh (\beta n),
$$

provided

$$
B= \pm A, \quad \lambda A^{2}=\alpha \tanh ^{2}(\beta / 2), \quad \beta v=4 \alpha \tanh (\beta / 2) .
$$

We find that this model also admits exact PT-invariant periodic solutions. Let us first note that Eq. (40) admits a periodic solution

$$
u_{n}=A \operatorname{dn}[\beta(n-v t), m],
$$

provided

$$
\lambda A^{2} \operatorname{cs}^{2}(\beta, m)=\alpha, \quad \beta v=\frac{2 \alpha}{\operatorname{cs}(\beta, m)} .
$$

Further, the same model (40) also admits another periodic solution

$$
u_{n}=A \sqrt{m} \operatorname{cn}[\beta(n-v t), m],
$$

provided

$$
\lambda A^{2} \mathrm{ds}^{2}(\beta, m)=\alpha, \quad \beta v=\frac{2 \alpha}{\operatorname{ds}(\beta, m)}
$$

We now show that the same model also admits a PT-invariant periodic solution

$$
u_{n}=A \operatorname{dn}[\beta(n-v t), m]+i B \sqrt{m} \operatorname{sn}[\beta(n-v t), m],
$$


provided

$$
B= \pm A, \quad \lambda A^{2}[\operatorname{cs}(\beta, m)+\mathrm{ns}(\beta, m)]^{2}=\alpha, \quad \beta v=\frac{4 \alpha \operatorname{sn}(\beta, m)}{1+\operatorname{cn}(\beta, m)}
$$

where $\operatorname{ns}(x, m)=1 / \operatorname{sn}(x, m)$. Further, the same model also admits another PT-invariant solution

$$
u_{n}=A \sqrt{m} \operatorname{cn}(\beta n, m)+i B \sqrt{m} \operatorname{sn}(\beta n, m),
$$

provided

$$
B= \pm A, \quad \lambda A^{2}[\operatorname{ds}(\beta, m)+\operatorname{ns}(\beta, m)]^{2}=\alpha, \quad \beta v=\frac{4 \alpha \operatorname{sn}(\beta, m)}{1+\operatorname{dn}(\beta, m)}
$$

\section{Solutions in Terms of $\operatorname{sech}^{2} x \pm i \operatorname{sech} x \tanh x$ and Periodic Generalization}

We now discuss two examples where $\operatorname{sech}^{2} x$ is a known solution and in both the cases we obtain new PT-invariant solutions in terms of $\operatorname{sech}^{2} x \pm i \operatorname{sech} x \tanh x$ and also PT-invariant periodic solutions in terms of $\mathrm{dn}^{2}(x, m) \pm i m \operatorname{sn}(x, m) \operatorname{cn}(x, m)$ as well as $\operatorname{dn}^{2}(x, m) \pm i \sqrt{m} \operatorname{sn}(x, m) \operatorname{dn}(x, m)$.

\subsection{KdV Equation}

We first discuss the celebrated KdV equation

$$
u_{t}+u_{x x x}-6 u u_{x}=0
$$

which is a well known integrable equation having application in several areas including shallow water waves [16]. It is also well known that it admits the soliton solution [16]

$$
u=A \operatorname{sech}^{2}(x-v t),
$$

provided $A=-2 \beta^{2}, v=4 \beta^{2}$. Remarkably, it also admits a PT-invariant solution

$$
u=A \operatorname{sech}^{2}(x-v t)+i B \operatorname{sech}(x-v t) \tanh (x-v t),
$$

provided

$$
B= \pm A, A=-\beta^{2}, v=\beta^{2} .
$$


We now show that KdV equation also admits periodic PT-invariant solutions. It is well known that one of the exact, periodic solutions to the KdV Eq. (53) is [16]

$$
u=A \operatorname{dn}^{2}[\beta(x-v t), m],
$$

provided

$$
A=-2 \beta^{2}, \quad v=4(2-m) \beta^{2} .
$$

Remarkably, even

$$
u=A \operatorname{dn}^{2}[\beta(x-v t), m]+i B m \operatorname{sn}[\beta(x-v t), m] \operatorname{cn}[\beta(x-v t), m],
$$

is an exact solution of the KdV Eq. (53) provided

$$
B= \pm A, \quad A=-\beta^{2}, \quad v=-(2-m) \beta^{2} .
$$

We thus have two new periodic solutions of the KdV Eq. (53) depending on whether $B=A$ or $B=-A$.

Remarkably, there is another PT-invariant solution to the same KdV equation

$$
u=A \operatorname{dn}^{2}\left[\beta\left(x-v t+\delta_{1}\right), m\right]+i B \sqrt{m} \operatorname{sn}[\beta(x-v t), m] \operatorname{dn}[\beta(x-v t), m]
$$

provided

$$
B= \pm A, \quad A=-\beta^{2}, \quad v=(5-4 m) \beta^{2} .
$$

Few remarks are in order at this stage.

1. It is well known that the hyperbolic potential $-2 \beta^{2} \operatorname{sech}^{2}(\beta x)$ which is a solution of the KdV equation, is a reflectionless potential. We then predict that the potentials $-\beta^{2} \operatorname{sech}^{2}(\beta x) \pm i \operatorname{sech}(\beta x) \tanh (x)$ must also be reflectionless potentials.

2. It is well known that the periodic potential $-2 \beta^{2} \operatorname{dn}^{2}(\beta x, m)$ which is a solution of the KdV equation, has precisely one band gap. We then predict that the potentials $-\beta^{2} \operatorname{dn}^{2}(\beta x, m) \pm i m \beta^{2} \operatorname{sn}(\beta x, m) \operatorname{cn}(\beta x, m)$ as well as the potentials $\beta^{2} \operatorname{dn}^{2}(\beta x, m) \pm i \sqrt{m} \beta^{2} \operatorname{sn}(\beta x, m) \operatorname{dn}(\beta x, m)$ must also have precisely one band gap. 


\section{$3.2 \quad \phi^{3}$ Field Theory}

This field theory arises in the context of third order phase transitions [21] and is also relevant to tachyon condensation [22]. The field equation for the $\phi^{2}-\phi^{3}$ field theory is given by

$$
\phi_{x x}=a \phi+b \phi^{2}
$$

which admits an exact solution

$$
\phi=A \operatorname{sech}^{2}(\beta x)+B
$$

provided

$$
A=-\frac{3 a}{2 b}, \quad \beta^{2}=\frac{a}{4}, \quad B=0 .
$$

Remarkably, Eq. (63) also admits a PT-invariant solution

$$
\phi=A \operatorname{sech}^{2}[\beta(x)] \pm i D \operatorname{sech}[\beta(x)] \tanh [\beta(x)]+B,
$$

provided

$$
D= \pm A, \quad A=-\frac{3 a}{b}, \quad \beta^{2}=a, \quad B=0 .
$$

Further the model also admits PT-invariant periodic solutions. Let us first note that the model (63) also admits the periodic solution

$$
\phi=\operatorname{Adn}^{2}[\beta(x), m]+B
$$

provided

$$
A=-\frac{3 a}{2 b \sqrt{1-m+m^{2}}}, \quad \beta^{2}=\frac{a}{4 \sqrt{1-m+m^{2}}}, \quad B=\frac{a\left[2-m-\sqrt{1-m+m^{2}}\right]}{2 b \sqrt{1-m+m^{2}}}
$$

It is easy to show that the same model also admits a PT-invariant periodic solution

$$
\phi=A \operatorname{dn}^{2}[\beta(x), m]+i D \sqrt{m} \operatorname{cn}[\beta(x), m] \operatorname{dn}[\beta(x), m]+B
$$

provided

$$
D= \pm A, \quad A=-\frac{3 a}{b \sqrt{16-16 m+m^{2}}}, \quad \beta^{2}=\frac{a}{\sqrt{16-16 m+m^{2}}}, \quad B=\frac{a\left[2-m-\sqrt{16-16 m+m^{2}}\right]}{2 b \sqrt{16-16 m+m^{2}}}
$$


Further, the same model also admits another PT-invariant periodic solution

$$
\phi=A \operatorname{dn}^{2}[\beta(x), m]+i D \sqrt{m} \operatorname{sn}[\beta(x), m] \operatorname{dn}[\beta(x+c), m]+B
$$

provided

$$
D= \pm A, \quad A=-\frac{3 a}{b \sqrt{1-16 m+16 m^{2}}}, \quad \beta^{2}=\frac{a}{\sqrt{1-16 m+16 m^{2}}}, \quad B=\frac{a\left[5-4 m-\sqrt{1-16 m+16 m^{2}}\right]}{2 b \sqrt{1-16 m+16 m^{2}}} .
$$

\section{PT-Invariant Solutions in Three Coupled models}

We now consider three different coupled models and show that in all these cases one has PT-invariant solutions for all the coupled fields.

\subsection{Coupled $\phi^{4}$ Model}

We first consider a coupled $\phi^{4}$ model

$$
\begin{aligned}
& \phi_{x x}=2 a_{1} \phi+4 b_{1} \phi^{3}+2 \gamma \phi \psi^{2}, \\
& \psi_{x x}=2 a_{2} \psi+4 b_{1} \psi^{3}+2 \gamma \psi \phi^{2},
\end{aligned}
$$

and show that even in this case, PT-invariant solutions are allowed in both the fields.

It is well known that this coupled system admits the solution [23]

$$
\phi=A \operatorname{sech}(\beta x), \quad \psi=D \operatorname{sech}(\beta x),
$$

provided

$$
2 b_{1} A^{2}+\gamma D^{2}=-\beta^{2}=2 b_{2} D^{2}+\gamma A^{2}, \quad a_{1}=a_{2}=\frac{\beta^{2}}{2} .
$$

Remarkably, the coupled model also admits the PT-invariant solution

$$
\begin{aligned}
& \phi=A \operatorname{sech}(\beta x)+i B \tanh (\beta x), \\
& \psi=D \operatorname{sech}(\beta x)+i F \tanh (\beta x),
\end{aligned}
$$


provided

$$
\begin{aligned}
& B= \pm A, \quad F= \pm D, \quad a_{1}=a_{2}=-\frac{\beta^{2}}{4} \\
& 4\left(2 b_{1} A^{2}+\gamma D^{2}\right)=-\beta^{2}=4\left(2 b_{2} D^{2}+\gamma A^{2}\right) .
\end{aligned}
$$

Note that the signs of $B= \pm A$ and $F= \pm D$ are correlated.

This coupled model also admits PT-invariant periodic solutions. Let us first note that one of the well known periodic solutions to the coupled Eq. (74) is [23]

$$
\phi=A \operatorname{dn}[\beta x, m], \quad \psi=D \operatorname{dn}[\beta x, m],
$$

provided

$$
2 b_{1} A^{2}+\gamma D^{2}=-\beta^{2}=2 b_{2} D^{2}+\gamma A^{2}, \quad a_{1}=a_{2}=\frac{(2-m) \beta^{2}}{2} .
$$

Further, the same coupled model is known to admit another periodic solution

$$
\phi=A \sqrt{m} \operatorname{cn}[\beta x, m], \quad \psi=D \sqrt{m} \operatorname{cn}[\beta x, m],
$$

provided

$$
2 b_{1} A^{2}+\gamma D^{2}=-\beta^{2}=2 b_{2} D^{2}+\gamma A^{2}, \quad a_{1}=a_{2}=\frac{(2 m-1) \beta^{2}}{2} .
$$

Remarkably, we find that the same coupled model also admits a PT-invariant periodic solution

$$
\begin{aligned}
& \phi=A \operatorname{dn}[\beta x, m]+i B \sqrt{m} \operatorname{sn}[\beta x, m], \\
& \psi=D \operatorname{dn}[\beta x, m]+i F \sqrt{m} \operatorname{sn}[\beta x, m],
\end{aligned}
$$

provided

$$
\begin{aligned}
& B= \pm A, \quad F= \pm D, \quad a_{1}=a_{2}=-\frac{(4 m-3) \beta^{2}}{4} \\
& 4\left(2 b_{1} A^{2}+\gamma D^{2}\right)=-\beta^{2}=4\left(2 b_{2} D^{2}+\gamma A^{2}\right) .
\end{aligned}
$$

Note that the signs of $B= \pm A$ and $F= \pm D$ are correlated.

Further, the same model also admits another PT-invariant periodic solution

$$
\begin{aligned}
& \phi=A \sqrt{m} \operatorname{cn}[\beta x, m]+i B \sqrt{m} \operatorname{sn}[\beta x, m], \\
& \psi=D \sqrt{m} \operatorname{cn}[\beta x, m]+i F \sqrt{m} \operatorname{sn}[\beta x, m],
\end{aligned}
$$


provided

$$
\begin{aligned}
& B= \pm A, \quad F= \pm D, \quad a_{1}=a_{2}=-\frac{(4-3 m) \beta^{2}}{4} \\
& 4\left(2 b_{1} A^{2}+\gamma D^{2}\right)=-\beta^{2}=4\left(2 b_{2} D^{2}+\gamma A^{2}\right) .
\end{aligned}
$$

Note that the signs of $B= \pm A$ and $F= \pm D$ are correlated.

\subsection{Coupled KdV Equations}

We now discuss the coupled KdV model which has also received some attention in the literature [24] and show that even in this case, PT-invariant solutions exist in both the coupled fields.

The coupled KdV equations are

$$
\begin{aligned}
& u_{t}+\alpha u u_{x}+\eta v v_{x}+u_{x x x}=0 \\
& v_{t}+\delta u v_{x}+v_{x x x}=0
\end{aligned}
$$

One of the well known solutions to the coupled Eqs. (88) is [24]

$$
u=A \operatorname{sech}^{2}[\beta(x-c t)], \quad v=D \operatorname{sech}^{2}[\beta(x-c t)],
$$

provided

$$
\delta A=12 \beta^{2}, \quad \eta D^{2}=(\delta-\alpha) A^{2}, \quad c=4 \beta^{2} .
$$

Remarkably, the same coupled model also admits the hyperbolic PT-invariant solution

$$
\begin{aligned}
& u=A \operatorname{sech}^{2}[\beta(x-c t)]+i B \tanh [\beta(x-c t)] \operatorname{sech}[\beta(x-c t)], \\
& v=D \operatorname{sech}^{2}[\beta(x-c t)]+i F \tanh [\beta(x-c t)] \operatorname{sech}[\beta(x-c t)],
\end{aligned}
$$

provided

$$
B= \pm A, \quad F= \pm D, \quad \delta A=6 \beta^{2}, \quad \eta D^{2}=(\delta-\alpha) A^{2}, \quad c=\beta^{2}
$$

This discussion is easily generalized to the periodic case. In particular, it is easy to check that the coupled Eqs. (88) have the periodic solution

$$
u=A \operatorname{dn}^{2}[\beta(x-c t), m], \quad v=D \operatorname{dn}^{2}[\beta(x-c t), m],
$$


provided

$$
\delta A=12 \beta^{2}, \quad \eta D^{2}=(\delta-\alpha) A^{2}, \quad c=4(2-m) \beta^{2} .
$$

Remarkably, the same model also admits a PT-invariant periodic solution

$$
\begin{aligned}
& u=A \operatorname{dn}^{2}[\beta(x-c t), m]+i B m \operatorname{sn}[\beta(x-c t), m] \operatorname{cn}[\beta(x-c t), m], \\
& v=D \operatorname{dn}^{2}[\beta(x-c t), m]+i F m \operatorname{sn}[\beta(x-c t), m] \operatorname{cn}[\beta(x-c t), m],
\end{aligned}
$$

provided

$$
B= \pm A, \quad F= \pm D, \quad \delta A=6 \beta^{2}, \quad \eta D^{2}=(\delta-\alpha) A^{2}, \quad c=(2-m) \beta^{2}
$$

Note that the signs of $B= \pm A$ and $F= \pm D$ are correlated. Further, the same model also admits another PT-invariant periodic solution

$$
\begin{aligned}
& u=A \operatorname{dn}^{2}[\beta(x-c t), m]+i B \sqrt{m} \operatorname{sn}[\beta(x-c t), m] \operatorname{dn}[\beta(x-c t), m], \\
& v=D \operatorname{dn}^{2}[\beta(x-c t), m]+i F \sqrt{m} \operatorname{sn}[\beta(x-c t), m] \operatorname{dn}[\beta(x-c t), m],
\end{aligned}
$$

provided

$$
B= \pm A, \quad F= \pm D, \quad \delta A=6 \beta^{2}, \quad \eta D^{2}=(\delta-\alpha) A^{2}, \quad c=(2 m-1) \beta^{2} .
$$

Note that the signs of $B= \pm A$ and $F= \pm D$ are correlated.

\subsection{Coupled KdV-mKdV Model}

Finally we consider a coupled $\mathrm{KdV}$-mKdV model [25]

$$
\begin{aligned}
& u_{t}+u_{x x x}+6 u u_{x}+2 \alpha u v v_{x}=0, \\
& v_{t}+v_{x x x}+6 v^{2} v_{x}+\gamma v u_{x}=0,
\end{aligned}
$$

and show that in this case too we have PT-invariant solutions of the form $\operatorname{sech}^{2} x \pm i \operatorname{sech} x \tanh x$ and $\operatorname{sech} x \pm i \tanh x$ in $\mathrm{KdV}$ and $\mathrm{mKdV}$ fields, $u$ and $v$, respectively.

It is easy to check that

$$
u=A \operatorname{sech}^{2}[\beta(x-c t)]+G, \quad v=D \operatorname{sech}[\beta(x-c t)],
$$


is an exact solution of the coupled Eqs. (99) provided

$$
12 D^{2}+4 \gamma A=12 \beta^{2}=6 A+\alpha D^{2}, \quad c=\beta^{2}, \quad G=-\frac{A}{4} .
$$

Remarkably, the same model also admits a PT-invariant solution

$$
\begin{aligned}
& u=A \operatorname{sech}^{2}[\beta(x-c t)]+i B \tanh [\beta(x-c t)] \operatorname{sech}[\beta(x-c t)], \\
& v=D \operatorname{sech}[\beta(x-c t)]+i F \tanh [\beta(x-c t)],
\end{aligned}
$$

provided

$$
B= \pm A, \quad F= \pm D, \quad 12 D^{2}+2 \gamma A=3 \beta^{2}=3 A+\alpha D^{2}, \quad c=-\frac{1}{2} \beta^{2}, \quad G=-\frac{A}{4} .
$$

We now show that the same model also has PT-invariant periodic solutions. Let us first note that

$$
u=A \operatorname{dn}^{2}[\beta(x-c t), m]+G, \quad v=D \operatorname{dn}[\beta(x-c t), m],
$$

is an exact solution of the coupled Eqs. (99) provided

$$
12 D^{2}+4 \gamma A=12 \beta^{2}=6 A+\alpha D^{2}, \quad c=(2-m) \beta^{2}, \quad G=-\frac{(2-m) A}{4} .
$$

It is easy to check that the same model also admits a PT-invariant solution

$$
\begin{aligned}
& u=A \operatorname{dn}^{2}[\beta(x-c t), m]+i B \sqrt{m} \operatorname{sn}[\beta(x-c t), m] \operatorname{dn}[\beta(x-c t), m]+G, \\
& v=D \operatorname{dn}[\beta(x-c t), m]+i F m \operatorname{sn}[\beta(x-c t), m]
\end{aligned}
$$

provided

$$
B= \pm A, \quad F= \pm D, \quad 12 D^{2}+2 \gamma A=3 \beta^{2}=3 A+\alpha D^{2}, \quad c=-\frac{(2 m-1)}{2} \beta^{2}, \quad G=-\frac{(3-2 m) A}{4} .
$$

Note that the signs of $B= \pm A$ and $F= \pm D$ are correlated. Further, the same model also admits another PT-invariant solution

$$
\begin{aligned}
& u=A \operatorname{dn}^{2}[\beta(x-c t), m]+i B m \operatorname{sn}[\beta(x-c t), m] \operatorname{cn}[\beta(x-c t), m], \\
& v=D \sqrt{m} \operatorname{cn}[\beta(x-c t), m]+i F \sqrt{m} \operatorname{sn}[\beta(x-c t), m] \operatorname{dn}[\beta(x-c t), m],
\end{aligned}
$$

provided

$$
B= \pm A, \quad F= \pm D, \quad 12 D^{2}+2 \gamma A=3 \beta^{2}=3 A+\alpha D^{2}, \quad c=-\frac{(2-m)}{2} \beta^{2}, \quad G=-\frac{(2-m) A}{4} .
$$

Note that the signs of $B= \pm A$ and $F= \pm D$ are correlated. 


\section{$5 \quad$ Summary and Conclusions}

In this paper we have shown through several examples that whenever a real nonlinear equation admits a solution in terms of $\operatorname{sech} x\left(\right.$ or $\left.\operatorname{sech}^{2} x\right)$, then the same model also admits solutions in terms of $\operatorname{sech} x \pm i \tanh x$ (or $\operatorname{sech}^{2} x \pm i \operatorname{sech} x \tanh x$ ). Further, we have also shown that such PT-invariant solutions also exist in the corresponding periodic case involving Jacobi elliptic functions.

The obvious open question is whether these results are true in general. It would be highly desirable if one can prove this in general, both in the hyperbolic as well as in the periodic case. In the absence of a general proof, it is worthwhile to look at additional examples and discern if this observation is true in general or if there are some exceptions. The other important question is: What could be the deeper underlying reason because of which such solutions exist? Another question is about the significance of such solutions for a real nonlinear equation. In this context we would like to remark that the symmetry of solutions of a nonlinear equation need not be the same as that of the nonlinear equation but it could be less.

Normally, the complex solutions of a real nonlinear equation are not of relevance. However, being PT-invariant complex solutions, we believe they could have some physical significance including in coupled optical waveguides $[1,10,14]$. One pointer in this direction is the fact that for both the $\mathrm{KdV}$ and the mKdV equations, which are integrable equations, we have checked that the first 3 constants of motion for the PT-invariant complex solutions of both the $\mathrm{KdV}$ and the $\mathrm{mKdV}$ equations are in fact real but have different values than those for the usual hyperbolic solution (and we suspect that in fact all the constants of motion would be real and would be different than those for the real hyperbolic solution) thereby suggesting that such solutions could be physically interesting. Thus it would be worthwhile to study the stability of such PT-invariant solutions, which may shed some light on the possible significance of such solutions. We hope to address some of these issues in the near future. 


\section{Acknowledgments}

One of us (AK) is grateful to B. Dey and P. Durga Nandini for stimulating discussions and to INSA for the award of INSA senior Scientist position at Savitribai Phule Pune University. This work was supported in part by the U.S. Department of Energy.

\section{References}

[1] See, e.g., S. H. H. Kachapi and D. D. Ganji, Nonlinear Equations: Analytical Methods and Applications, (Springer, Berlin, 2016).

[2] See, e.g., C. Rogers and W. K. Schief, Bäcklund and Darboux transformations, (Cambridge Univ. Press, 2002).

[3] M. Jia, F. Huang, and S. Y. Lou, Phys. Scr. 73 (2006) 368.

[4] A. Khare and A. Saxena, Phys. Lett. A 377 (2013) 2761.

[5] A. Khare and A. Saxena, J. Math. Phys. 55 (2014) 032701.

[6] See, e.g., M. Abromowitz and I.A. Stegun, Handbook of Mathematical Functions (Dover Publications, New York, 2010).

[7] See, e.g., C. M. Bender, Rep. Prog. Phys. 70 (2007) 947 and references therein.

[8] See, e.g., J. Schindler, Z. Lin, M. C. Zheng, F. M. Ellis, and T. Kottos, Phys. Rev. A 84 (2011) 040101; J. Schindler, Z. Lin, J. M. Lee, H. Ramezani, F. M. Ellis, and T. Kottos, J. Phys. A: Math. Theor. 45 (2012) 444029.

[9] C. M. Bender, B. Berntson, D. Parker, and E. Samuel, Am. J. Phys. 81 (2013) 173.

[10] B. Peng, S. K Özdemir, F. Lei, F. Monifi, M. Gianfreda, G. L. Long, S. Fan, F. Nori, C. M. Bender, and L. Young, Nature Phys. 10 (2014) 394. 
[11] K. G. Makris, R. El-Ganainy, D. N. Christodoulides, and Z. H. Musslimani, Int. J. Theor. Phys. 50 (2011) 1019 .

[12] P. G. Kevrekidis, J. Cuevas-Maraver, A. Saxena, F. Cooper, and A. Khare, Phys. Rev. E 92 (2015) 042901.

[13] J. Cuevas-Maraver, P. G. Kevrekidis, A. Saxena, F. Cooper, A. Khare, A. Comech, and CM Bender, IEEE: J. Selected Topics Quant. Electron. 22 (2015) 1; arXiv:1508.00852.

[14] C. E. Rüter, K. G. Makris, R. El-Ganainy, D. N. Christodoulides, M. Segev, and D. Kip, Nat. Phys. 6, $192(2010)$.

[15] See for example, C.M. Bender, D.C. Brody, J.H. Chen and E. Furlan, J. Phys. A: Math. and Theor. 40 (2007) F153; A. Fring, ibid 40 (2007) 4215; C.M. Bender, F. Cooper, A. Khare, B. Mihaila and A. Saxena, Pramana 73 (2009) 375.

[16] See, e.g., Solitons: An Introduction by P. G. Drazin and R. S. Johnson, (Cambridge Univ. Press. 1989) and references therein.

[17] S. Aubry, J. Chem. Phys. 64 (1976) 3392.

[18] F. Cooper, A. Khare, B. Mihaila, and A. Saxena, Phys. Rev. E 72 (2005) 036605.

[19] A. Khare and U.P. Sukhatme, J. Math. Phys. 43 (2002) 3798; A. Khare, A. Lakshminarayan and U.P. Sukhatme, ibid 44 (2003) 1822; Pramana 62 (2004) 1201.

[20] Y. Zhang, J. Mei, and Y. C. Hon, Phys. Essays 23 (2010) 276.

[21] P. Kumar, D. Hall, and R.G. Goodrich, Phys. Rev. Lett. 82 (1999) 4532; P. Kumar and A. Saxena, Phil. Mag. B 82 (2002) 1201.

[22] B. Zwiebach, J. High Energy Phys. 09 (2000) 028; J.A. Minahan and B. Zwiebach, ibid 09 (2000) 029.

[23] A. Khare and A. Saxena, J. Math. Phys. 47 (2006) 092902. 
[24] Y. Zhou, M. Wang, and Y. Wang, Phys. Lett. A 308 (2003) 31.

[25] A. A. Halim and S. B. Leble, Chaos, Solitons \& Fractals 19 (2004) 99. 